\title{
PFPRINTE

\section{8) Multimorbidade em idosas de um centro de referência e atenção ao idoso}

\author{
Luísa Victória Biasi'; Ana Carolina Bertoletti De Marchi²; Ana Luisa Sant'Anna Alves²; \\ Natália Aresi Ceolin²; Tainá Bressan Giacomini²; Valéria Paula Selong²; Juliane Bervian²; \\ Marlene Doring'2; Marilene Rodrigues Portella²; Helenice de Moura Scortegagna²; \\ Bernadete Maria Dalmolin²
}

\section{Resumo}

Introdução: A multimorbilidade é definida como a presença de duas ou mais doenças crônicas (DC) e está associada à diminuição da qualidade de vida. Objetivo: Identificar a presença de multimorbidade em idosas. Metodologia: Estudo transversal com idosas, com 60 anos ou mais, matriculadas em oficinas do Centro de Referência e Atenção ao Idoso da Universidade de Passo Fundo/RS (Creati-UPF). Foi aplicado um questionário contendo variáveis demográficas, socioeconômicas e presença de patologias. Resultados: Foram investigadas 340 idosas, dessas 43,2\% apresentavam duas ou mais DC. A prevalência foi maior entre as idosas da classe econômica C, em relação as classes A e B, em idosas com sobrepeso e obesidade quando comparado com as idosas com baixo peso e eutróficas. Conclusões: A partir dos resultados obtidos, este achado pode contribuir para o manejo de idosas com multimorbidade.

Palavras-chave: Idosas; envelhecimento; doenças crônicas não transmissíveis.

\section{A multimorbidade em idosos}

A multimorbidade é considerada como o acontecimento de duas ou mais doenças crônicas (DC) físicas ou mentais, de forma paralela em um indivíduo. Pessoas que se encontram nessa situação, principalmente idosos, são propensos a passar por um maior número de hospitalizações, com isso utilizam simultaneamente muitos medicamentos, aumentando assim a vulnerabilidade aos efeitos adversos (LEFÈVRE et al, 2014). Nas últimas décadas o envelhecimento populacional, se configura como um dos maiores fenômenos demográficos, o que se apresenta como um alerta à necessidade de uma maior atenção a essa questão, uma vez

Programa de Pós-Graduação em Envelhecimento Humano, Faculdade de Educação Física e Fisioterapia. Endereço de correspondência: Universidade de Passo Fundo / BR 285, São José I Passo Fundo/RS I CEP: 99052-900/ Brasil. E-mail: luisavicc@gmail.com

2 Professores do Programa de Pós-Graduação Stricto Sensu em Envelhecimento Humano, Faculdade de Educação Física e Fisioterapia, Universidade de Passo Fundo. E-mails: carolina@upf.br; alves.als@upf.br; doring@upf.br; portella@upf.br 
que um dos principais problemas desse processo é a alta prevalência de DC que podem causar prejuízo na mobilidade e na autonomia do portador, levando a consequências como quedas e deficiências (OMS, 2003; BARNETT et al, 2012). O número de morbidades e a proporção de pessoas com multimorbidade aumenta consideravelmente com a idade. Atualmente, metade da população com 50 anos possui pelo menos uma morbidade e indivíduos com 65 anos, em sua maioria, é multimórbido. Já aos 85 anos, a prevalência corresponde a $82 \%$. Entretanto, apesar de aumentar a prevalência conforme a idade, as áreas socialmente desfavorecidas não as principais acometidas. Adultos que residem em áreas mais carentes possuem multimorbidade equivalente àquele com idade entre 10 a 15 anos mais velhos. Além disso, a presença da desordem mental é comum em pessoas que possuem transtornos físicos. A comorbidade de saúde física e mental é mais prevalente em mulheres do que homens, e muito maior em todos os aspectos, em idosos (BARNETT et al, 2012). Com isso, fica claro que a multimorbidade está significativamente associada a maior idade, sexo feminino, nível socioeconômico baixo e presença de transtornos mentais (VIOLAN et al, 2014). As doenças crônicas não transmissíveis (DCNT) que mais acometem indivíduos de 60 anos ou mais estão principalmente relacionadas ao sistema cardiovascular, como a Hipertensão Arterial Sistêmicas (HAS) e Acidente Vascular Encefálico (AVE), resultando num acréscimo nas taxas de glicose, podendo ocasionar Diabetes Mellitus (DM) e Câncer ${ }^{5}$. Juntamente com a presença dessas doenças, o estado nutricional vulnerável, identificado pelo índice de massa corporal é comum entre os idosos acometidos pelas DCNT (FARES et al 2012). O excesso de peso e a desnutrição são fatores que estão associados à multimorbidade nesses indivíduos (DIXON et al, 2015). Tendo em vista as possíveis relações entre DC e o estado nutricional dos idosos, o uso de indicadores clínicos e antropométricos passaram a ser utilizados para auxiliar na identificação de fatores de risco de multimorbidade, sendo primordiais as medidas de peso e altura para o cálculo de Índice de Massa Corporal (IMC), método de baixo custo e fácil aplicação, possibilitando significativa confiança na determinação da gordura corporal (COSTA, 2013). Neto, Barbosa, Meneghini (2016) avaliaram a multimorbidade e o índice de massa corporal associado à doenças e condições crônicas de saúde. Em sua pesquisa, avaliaram 477 indivíduos acima de 60 anos e encontraram diferenças significativas entre homens e mulheres na prevalência estimada de doenças e condições crônicas de saúde. Da mesma maneira, a associação com o índice de massa corporal (IMC) também diferiu entre os sexos. Nas mulheres, a hipertensão foi associada com maior IMC, ou seja, obesidade, e doença pulmonar crônica foi associada a valores mais baixos de IMC, ou seja, baixo peso. Dados da Pesquisa Nacional por Amostra de Domicílios (PNAD) de 2008 mostrou que 5,9\% da população relata ter três ou mais doenças crônicas e a proporção aumenta com a idade. Entre os idosos, 79,1\% relataram ter pelo menos uma doença crônica, $15,2 \%$ relataram restrições nas atividades habituais e cerca de $12 \%$ relataramhistórico de internações nos últimos 12 meses (IBGE, 2008). No Rio Grande do Sul, mais especificadamente em municípios de pequeno porte do norte do estado, foi realizado um estudo com idosos residentes nessas cidades, que avaliou a multimorbidade associado à autopercepção negativa de saúde obteve um resultado muito semelhante no que diz respeito à multimorbidade, em que houve uma prevalência de $45 \%$ dos indivíduos que possuem essa patologia (CAVALCANTI, 2017). Outro estudo realizado no Acre, que avaliou a multimorbidade em idosos atendidos 
pela Estratégia da Saúde da Família, com uma totalidade de 264 idosos avaliados, obteve uma prevalência maior de multimorbidade do que o presente estudo, sendo de 66,3\% (2018). A partir dos resultados parciais de um projeto de pesquisa e extensão universitária da Universidade Federal de Campina Grande (PB), outro estudo relacionado à multimorbidades foi realizado, que teve como base o questionário da Pesquisa Nacional de Saúde (PNS) 2013 (IBGE, 2013). Nesse estudo, 83,3\% dos entrevistados apresentam multimorbidades, apontando uma maior prevalência dessas em pessoas com obesidade e sobrepeso $(66,7 \%)$ e em pessoas com baixa classe socioeconômica (SALIVE, 2013; CARVALHO et al, 2017). A partir disso, o presente estudo tem como objetivo identificar a presença de multimorbidade em idosas.

\section{Metodologia}

Trata-se de um estudo transversal com idosas de 60 anos ou mais, matriculadas em oficinas do Centro de Referência e Atenção ao Idoso da Universidade de Passo Fundo/RS (Creati-UPF). Este estudo faz parte do projeto "Estudo Longitudinal do Centro de Referência e Atenção ao Idoso: ELO-Creati”, aprovado pelo Comitê de Ética em Pesquisa da Universidade de Passo Fundo sob parecer no $\mathbf{7 4 1 . 2 1 4}$, destaca-se que todos os indivíduos foram preservados através do Termo de Consentimento Livre e Esclarecido. Para responder aos objetivos do estudo foi aplicado um questionário contendo variáveis demográficas e socioeconômicas, além de presença de doenças.

\section{Resultados/conclusões}

Foram investigadas 340 idosas de 60 a 89 anos (média 69,05 anos DP=0,372). Quanto à multimorbidade, $43,2 \%$ apresentavam duas ou mais doenças crônicas. A prevalência de multimorbidade foi maior entre as idosas pertencentes a classe econômica C (49,6\%) quando comparado as classes econômicas A e B $(38,0 \%)$ ( $p=0,025)$, em idosas com sobrepeso e obesidade $(55,1 \%)$ quando comparado com as idosas com baixo peso $(36 \%)$ e eutróficas $(39,3 \%)$ $(\mathrm{p}=0,022)$. As idosas ativas apresentaram menor prevalência de multimorbidade $(28,2 \%) \mathrm{em}$ relação às idosas insuficientemente ativas $(47,6 \%)$ e suficientemente ativas $(43,6 \%)$, no entanto, não houve associação significativa. Ao final desse estudo, é possível perceber a existência de uma maior prevalência de multimorbidade em idosas da classe econômica $\mathrm{C}$ e com excesso de peso e obesidade. A partir desses resultados, e frente ao progressivo envelhecimento da população, conclui-se que o idoso com multimorbidade merece atenção especial, havendo uma necessidade de intervenções na saúde em diversos aspectos, não apenas para tratar as doenças crônicas, mas com o principal objetivo de promover a melhoria da qualidade de vida.

\section{Reconhecimento e agradecimentos}

O presente trabalho foi realizado com apoio da Coordenação de Aperfeiçoamento de Pessoal de Nível Superior - Brasil (CAPES) - Código de Financiamento 001 


\section{The multimorbity in the elderly of a reference center and the attention to the elderly}

\section{Abstract}

Introduction: Multimorbidity is defined as the presence of two or more chronic diseases (CD) and is associated with decreased quality of life. Objective: To identify the presence of multimorbity in the elderly. Methodology: Cross-sectional study over 60 years, enrolled in workshops at the Center for Reference and Elderly Care of the University of Passo Fundo / RS (Creati-UPF). It was anchored with demographic, socioeconomic variables and presence of pathologies. Results: We investigated 340 elderly women, $43.2 \%$ with the initials of two or more DCs. The difference is between the economic economy classes $C$, in relation to the classes $\mathrm{A}$ and $\mathrm{B}$, in comparison with the obesity when compared with the classes with low weight and eutrophic. Conclusions: Based on the results obtained, this finding may contribute to the management of elderly women with multimorbity.

Keywords: Elderly. Aging. Noncommunicable chronic diseases.

\section{Referências}

AMARAL T.L.M., et al. Multimorbidade, depressão e qualidade de vida em idosos atendidos pela Estratégia de Saúde da Família em Senador Guiomard, Acre, Brasil. Ciênc. saúde coletiva vol.23 no.9 Rio de Janeiro set. 2018.

BARNETT, K, et al. Epidemiology of multimorbidity and implications for health care, research, and medical education: a cross-sectional study. The Lancet, United Kingdom, v. 380, n. 9836, p. 37-43, 2012.

CARVALHO, J.N, et al. Prevalence of multimorbidity in the Brazilian adult population according to socioeconomic and demographic characteristics. PLoS One. 2017;12(4):1-13.

CAVALCANTI, et al. Multimorbidade associado à polifarmácia e autopercepção negativa de saúde. Rev. Bras. Geriatr. Gerontol., Rio de Janeiro, 2017; 20(5): 635-643.

COSTA, A.G.S. et al. Ocorrência de quedas e Índice de Massa Corporal em Idosos. Rev. enferm. UERJ, 2013.

DIXON, J.B. et al. 'Obesity paradox' misunderstands the biology of optimal weight throughout the life cycle, Int J Obes. 39(1):82-4, 2015.

FARES, D. et al. Fatores associados ao estado nutricional de idosos de duas regióes do Brasil. Rev Assoc Med Bras.,58(4):434-41, 2012

IBGE. Instituto Brasileiro de Geografia e Estatística (IBGE). Pesquisa Nacional de Saúde 2013/2014. 181 p.

IBGE. Instituto Brasileiro de Geografia e Estatística. Pesquisa Nacional por Amostra de Domicílios - um panorama da Saúde no Brasil: acesso e utilização dos serviços, condições de saúde e fatores de risco e proteção à saúde (PNAD 2008): Rio de Janeiro. 2010; Disponível em: http://www.ibge.gov.br/home/estatistica/populacao/ panorama_saude_brasil_2003_2008/ [2018 set 26].

LEFÈVRE, T. et al. What do we mean by multimorbidity? an analysis of the literature on multimorbidity measures, associated factors, and impact on health services organization. Rev Epidemiol Santé Publique. 2014;62(5):305-14.

MINISTÉRIO DA SAÚDE. Plano de Ações Estratégicas para o Enfrentamento das Doenças Crônicas Não Transmissíveis (DCNT) no Brasil 2011-2022 [Internet]. Brasília, DF: MS; 2011. Disponível em: http:// bvsms.saude.gov.br/bvs/publicacoes/plano_acoes_enfrent_dent_2011.pdf.

NETO J.S.L., BARBOSA A.R., MENEGHINI V. Doenças e condições crônicas de saúde, multimorbidade e índice de massa corporal em idosos. Rev. bras. cineantropom. desempenho hum. vol.18 no.5 Florianópolis set./out. 2016. 
OMS. Organização Mundial da Saúde. Cuidados inovadores para condições crônicas: componentes estruturais de ação: relatório mundial: Brasília; 2003; Available from: http://whqlibdoc.who.int/hq/2002/WHO_NMC_ CCH_02.01_por.pdf [2013 dez 16].

SALIVE M.E. Multimorbidity in older adults. Epidemiol Rev. 2013;1(1):75-83.

VIOLAN, C. et al. Prevalence, Determinants and Patterns of Multimorbidity in Primary Care: A Systematic Review of Observational Studies. Plos One, v. 9, 2014. 